PROCEEDINGS OF THE

AMERICAN MATHEMATICAL SOCIETY

Volume 133, Number 6, Pages 1829-1835

S 0002-9939(05)07719-1

Article electronically published on January 25, 2005

\title{
A NEW PROXIMAL POINT ITERATION THAT CONVERGES WEAKLY BUT NOT IN NORM
}

\author{
H. H. BAUSCHKE, J. V. BURKE, F. R. DEUTSCH, H. S. HUNDAL, \\ AND J. D. VANDERWERFF \\ (Communicated by Jonathan M. Borwein)
}

\begin{abstract}
In 1991, Güler constructed a proximal point iteration that converges weakly but not in norm. By building on a recent result of Hundal, we present a new, considerably simpler, example of this type.
\end{abstract}

\section{INTRODUCTION}

Throughout, $X$ denotes a real Hilbert space with inner product $\langle\cdot, \cdot\rangle$ and induced norm $\|\cdot\|$. Let $\varphi: X \rightarrow]-\infty,+\infty]$ be a convex, lower semicontinuous, and proper function. The Proximal Point Algorithm, first introduced by Martinet [1] in 1970 (see also [15]), is a now-classical method for solving the convex minimization problem

$$
\min _{x \in X} \varphi(x)
$$

iteratively: given a starting point $x_{0}$ and parameter sequence $\left(\gamma_{n}\right)_{n \in \mathbb{N}}$ of strictly positive reals, a sequence is generated by

$$
(\forall n \in \mathbb{N}=\{0,1,2, \ldots,\}) \quad x_{n+1}=\underset{x \in X}{\arg \min }\left[\varphi(x)+\frac{1}{2 \gamma_{n}}\left\|x-x_{n}\right\|^{2}\right] .
$$

Brézis and Lions [7] proved that if $\varphi$ has minimizers and $\sum_{n \in \mathbb{N}} \gamma_{n}=+\infty$, then the sequence $\left(x_{n}\right)_{n \in \mathbb{N}}$ generated by (1.2) converges weakly to a solution of (1.1). The question whether the convergence of such a sequence is always in norm was answered in the negative by Güler [9]. His counterexample is quite complicated and relies on several deeper results about nonlinear contraction semigroups. (See 4] and [17] for recent modifications of the Proximal Point Algorithm that produce norm convergent sequences.)

The aim of this paper is to provide a new, conceptually simple, proximal point iteration that converges weakly but not in norm.

Received by the editors September 13, 2002.

2000 Mathematics Subject Classification. Primary 65K10, 90C25; Secondary 47H05, 47H09, $65 \mathrm{~K} 05$.

Key words and phrases. Proximal point algorithm, weak convergence, support point.

The first author was supported in part by the Natural Sciences and Engineering Research Council of Canada. The second author was supported in part by the National Science Foundation Grant DMS-0203175. The third author was supported in part by the National Science Foundation Grant DMS-0204569. 
The paper is organized as follows. In Section 2 we establish the basic convergence theory of the Support Point Algorithm for finding points in a closed convex set supported by a functional. The convergence results resemble those known for the Proximal Point Algorithm, and this is not a coincidence: in fact, in Section 3 it is shown that the Support Point Algorithm is a special case of the Proximal Point Algorithm. Hundal's recent example [10] of an alternating projections iteration that converges weakly but not in norm is discussed in Section 4. In the final Section 5 . we show that Hundal's example can be viewed as an instance of the Support Point Algorithm. Altogether, we have obtained a sequence generated by the Proximal Point Algorithm that is weakly convergent, but not in norm.

We employ standard notation and terminology from Convex Analysis; see, e.g., [14] or [16]. Specifically, $I$ stands for the identity operator, and given a closed convex set $C, \iota_{C}$ denotes the indicator function of a set $C$ in $X\left(\iota_{C}(x)=0\right.$, if $x \in C ; \iota_{C}(x)=+\infty$, otherwise); and $N_{C}$ is the normal cone map $\partial \iota_{C}\left(N_{C}(x)=\right.$ $\left\{x^{*} \in X: \sup \left\langle x^{*}, C-x\right\rangle=0\right\}$, if $x \in C ; N_{C}(x)=\varnothing$, otherwise).

\section{Convergence theory of the Support Point Algorithm}

This section deals with the Support Point Algorithm, which is an iterative method for finding support points. It was analyzed by Bauschke and Vanderwerff in 1996 [1, Section 6.3.2]. The Support Point Algorithm will later be exhibited as an incarnation of the Proximal Point Algorithm. Thus, its convergence properties could be deduced from known results on the convergence of the Proximal Point Algorithm; however, to make the paper more self-contained and because the proofs for the Support Point Algorithm are short and instructive, we include the details.

Definition 2.1 (support points). Suppose $C$ is a closed convex nonempty set in $X$ and $f \in X \backslash\{0\}$. Then the set of support points of $C$ with respect to $f$ [14] is

$$
\operatorname{supp}(f, C)=\{x \in C:\langle f, x\rangle=\sup \langle f, C\rangle\}=\underset{x \in C}{\arg \max } f(x),
$$

where $f(x)=\langle f, x\rangle$.

We will introduce an iterative method for finding support points, which employs the projector onto $C$.

Definition 2.2 (projector and distance). Suppose $C$ is a closed convex nonempty set in $X$. Then for every $x \in X$, there exists a unique point $P_{C} x \in C$ nearest to $x$ : $\left\|P_{C} x-x\right\|=\min _{c \in C}\|c-x\|$. The induced nearest point map $P_{C}: X \rightarrow C$ is called the projector onto $C$, and the function $d(\cdot, C): X \rightarrow \mathbb{R}: x \mapsto\left\|x-P_{C} x\right\|$ measures the distance to the set $C$.

The following two results are well known.

Proposition 2.3 (basic properties). Suppose $C$ is a closed convex nonempty set in $X$. Then:

(i) $(\forall x \in X) P_{C} x$ is characterized by $P_{C} x \in C$ and $\sup \left\langle C-P_{C} x, x-P_{C} x\right\rangle=0$.

(ii) $P_{C}$ is firmly nonexpansive, i.e.,

$(\forall x \in X)(\forall y \in X) \quad\left\|P_{C} x-P_{C} y\right\|^{2}+\left\|\left(I-P_{C}\right) x-\left(I-P_{C}\right) y\right\|^{2} \leq\|x-y\|^{2}$.

(iii) $P_{C}=\left(I+N_{C}\right)^{-1}$ 
Proof. (i): [8, Section 4.1]. (ii): 8, Section 5.5]. (iii): Pick $x \in X$ and let $y=P_{C} x$. By (i), $y \in C$ and $\sup \langle C-y, x-y\rangle=0$. Hence $x-y \in N_{C}(y) \Leftrightarrow x \in\left(I+N_{C}\right)(y)$ $\Leftrightarrow y \in\left(I+N_{C}\right)^{-1}(x)$.

Proposition 2.4 (support points and the projector). Suppose $C$ is a closed convex nonempty set in $X, f \in X$ such that $\|f\|=1$, and $x \in X$. Then:

(i) The following are equivalent:

(a) $x \in \operatorname{supp}(f, C)$;

(b) $f \in N_{C}(x)$;

(c) $(\forall \alpha>0) P_{C}(x+\alpha f)=x$;

(d) $(\exists \alpha>0) P_{C}(x+\alpha f)=x$.

(ii) $(\forall \alpha>0)(\forall c \in C)$

$\left\|P_{C}(x+\alpha f)-c\right\|^{2} \leq\|x-c\|^{2}-\left\|x-P_{C}(x+\alpha f)\right\|^{2}+2 \alpha\langle f, x-c\rangle+2 \alpha\left\langle f, P_{C}(x+\alpha f)-x\right\rangle$.

Proof. (See also 1, Proposition 6.3.1 and Proposition 6.3.2].) (i): Fix $\alpha>0$. Then $x \in \operatorname{supp}(f, C) \Leftrightarrow x \in C$ and $\langle f, x\rangle=\sup \langle f, C\rangle \Leftrightarrow x \in C$ and $\langle\alpha f, x\rangle=$ $\sup \langle\alpha f, C\rangle \Leftrightarrow x \in C$ and $\sup \langle C-x,(x+\alpha f)-x\rangle=0 \Leftrightarrow x=P_{C}(x+\alpha f)$ (by Proposition 2.3. (i)) $\Leftrightarrow x=\left(I+N_{C}\right)^{-1}(x+\alpha f)$ (by Proposition 2.3. (iii)) $\Leftrightarrow x+\alpha f \in$ $\left(I+N_{C}\right)(x)=x+N_{C}(x) \Leftrightarrow \alpha f \in N_{C}(x) \Leftrightarrow f \in N_{C}(x)$. The equivalences follow. (ii): By Proposition 2.3(ii) (applied to $c$ and $x+\alpha f$ ), we have $\|c-(x+\alpha f)\|^{2} \geq$ $\left\|c-P_{C}(x+\alpha f)\right\|^{2}+\left\|(x+\alpha f)-P_{C}(x+\alpha f)\right\|^{2}$. Thus, using $\|f\|=1$,

$$
\begin{aligned}
\left\|c-P_{C}(x+\alpha f)\right\|^{2} \leq & \|c-(x+\alpha f)\|^{2}-\left\|(x+\alpha f)-P_{C}(x+\alpha f)\right\|^{2} \\
= & \|(x-c)+\alpha f\|^{2}-\left\|\left(x-P_{C}(x+\alpha f)\right)+\alpha f\right\|^{2} \\
= & \|x-c\|^{2}+\alpha^{2}+2\langle x-c, \alpha f\rangle-\left\|x-P_{C}(x+\alpha f)\right\|^{2} \\
& -\alpha^{2}-2\left\langle x-P_{C}(x+\alpha f), \alpha f\right\rangle .
\end{aligned}
$$

The proof is complete.

Theorem 2.5 (Support Point Algorithm). Suppose $C$ is a closed convex set in $X$ and $f \in X$ such that $\|f\|=1$. Let $\left(\alpha_{n}\right)_{n \in \mathbb{N}}$ be a sequence of strictly positive parameters, fix a starting point $x_{0} \in C$, and generate a sequence $\left(x_{n}\right)_{n \in \mathbb{N}}$ by

$$
(\forall n \in \mathbb{N}) \quad x_{n+1}=P_{C}\left(x_{n}+\alpha_{n} f\right) .
$$

We refer to this iterative method as the Support Point Algorithm with respect to $f$ and $C$, with starting point $x_{0}$ and parameter sequence $\left(\alpha_{n}\right)_{n \in \mathbb{N}}$.

(i) If $\sum_{n \in \mathbb{N}} \alpha_{n}<+\infty$, then $\left(x_{n}\right)_{n \in \mathbb{N}}$ converges in norm to some point in $C$.

(ii) If $\sum_{n \in \mathbb{N}} \alpha_{n}=+\infty$, then the following dichotomy holds.

Either $\operatorname{supp}(f, C) \neq \varnothing$ and $\left(x_{n}\right)_{n \in \mathbb{N}}$ converges weakly to some point in $\operatorname{supp}(f, C)$, or $\operatorname{supp}(f, C)=\varnothing$ and $\left\|x_{n}\right\| \rightarrow+\infty$.

Proof. Observe that the sequence $\left(x_{n}\right)_{n \in \mathbb{N}}$ lies entirely in $C$.

(i): Fix $n \in \mathbb{N}$. By Proposition 2.3], (ii), $\left\|x_{n+1}-x_{n}\right\|=\left\|P_{C}\left(x_{n}+\alpha_{n} f\right)-P_{C} x_{n}\right\| \leq$ $\left\|\left(x_{n}+\alpha_{n} f\right)-x_{n}\right\|=\alpha_{n}$; consequently, $\sum_{n \in \mathbb{N}}\left\|x_{n+1}-x_{n}\right\| \leq \sum_{n \in \mathbb{N}} \alpha_{n}<+\infty$. Hence $\left(x_{n}\right)_{n \in \mathbb{N}}$ is a Cauchy sequence, and it must therefore converge to some point in $C$.

(ii): (See also 1, Theorem 6.3.3].) For brevity, set $S=\operatorname{supp}(f, C)$.

Claim 1: $\left(f\left(x_{n}\right)\right)_{n \in \mathbb{N}}$ is increasing.

Indeed, by Proposition 2.3 (i), $0 \geq\left\langle x_{n}-x_{n+1},\left(x_{n}+\alpha_{n} f\right)-x_{n+1}\right\rangle=$ $\left\|x_{n}-x_{n+1}\right\|^{2}+\alpha_{n} f\left(x_{n}-x_{n+1}\right)$. 
Claim 2: $\left.\left.\lim _{n} f\left(x_{n}\right)=\sup f(C) \in\right]-\infty,+\infty\right]$.

By Claim 1, the limit of $\left(f\left(x_{n}\right)\right)_{n \in \mathbb{N}}$ exists in $\left.]-\infty,+\infty\right]$. It suffices to show that for every $c \in C$ and all $\varepsilon>0$, eventually $f\left(x_{n}\right)>f(c)-\varepsilon$. Suppose this were not the case. Then there exist $c \in C$ and $\varepsilon>0$ such that $(\forall n \in \mathbb{N}) f\left(x_{n}\right) \leq f(c)-\varepsilon$. By Proposition 2.4(ii),

$$
\begin{aligned}
\left\|x_{n+1}-c\right\|^{2} & \leq\left\|x_{n}-c\right\|^{2}-\left\|x_{n}-x_{n+1}\right\|^{2}+2 \alpha_{n}(-\varepsilon)+2 \alpha_{n}\left\langle f, x_{n+1}-x_{n}\right\rangle \\
& \leq\left\|x_{n}-c\right\|^{2}+2 \alpha_{n}\left(\left\langle f, x_{n+1}-x_{n}\right\rangle-\varepsilon\right) .
\end{aligned}
$$

Now as $\left(f\left(x_{n}\right)\right)_{n \in \mathbb{N}}$ converges to a finite limit, eventually $\left\langle f, x_{n+1}-x_{n}\right\rangle \leq \varepsilon / 2$. Thus there exists $\bar{n} \in \mathbb{N}$ such that $(\forall n \geq \bar{n})\left\|x_{n+1}-c\right\|^{2}-\left\|x_{n}-c\right\|^{2} \leq 2 \alpha_{n}(-\varepsilon / 2)$. This implies $-\left\|x_{\bar{n}}-c\right\|^{2} \leq-\varepsilon \sum_{n \geq \bar{n}} \alpha_{n}=-\infty$, which is absurd. Claim 2 is verified.

Claim 3: If $S=\varnothing$, then $\left\|x_{n}\right\| \stackrel{+\infty}{\rightarrow}$.

Assume the opposite. Then some subsequence of $\left(x_{n}\right)_{n \in \mathbb{N}}$ is bounded and, in view of Claim 2, its weak cluster points would lie in $S$, which is absurd. Claim 3 thus holds.

Claim 4: If $S \neq \varnothing$, then $\left(x_{n}\right)_{n \in \mathbb{N}}$ converges weakly to some point in $S$.

It is readily verified that $S$ is a closed convex set; moreover, if $\alpha>0$, then Proposition 2.4. (i) shows that $s \in S$ if and only if $s=P_{C}(s+\alpha f)$. Using Proposition 2.3 (ii), we deduce

$$
\begin{aligned}
(\forall s \in S)(\forall n \in \mathbb{N}) \quad\left\|x_{n+1}-s\right\| & =\left\|P_{C}\left(x_{n}+\alpha_{n} f\right)-P_{C}\left(s+\alpha_{n} f\right)\right\| \\
& \leq\left\|\left(x_{n}+\alpha_{n} f\right)-\left(s+\alpha_{n} f\right)\right\| \\
& =\left\|x_{n}-s\right\| .
\end{aligned}
$$

Consequently, $\left(x_{n}\right)_{n \in \mathbb{N}}$ is Fejér monotone with respect to $S$. On the other hand, Claim 2 shows that all weak cluster points of $\left(x_{n}\right)_{n \in \mathbb{N}}$ must lie in $S$. Altogether, by [2, Theorem 2.16], the sequence $\left(x_{n}\right)_{n \in \mathbb{N}}$ converges weakly to some point in $S$.

In view of Claim 3 and Claim 4, the entire theorem is proven.

\section{Support Point Algorithm as a Proximal Point Algorithm}

Assume that $\varphi: X \rightarrow]-\infty,+\infty]$ is a convex, lower semicontinuous, proper function. Recall that (see (1.2)), given a sequence of strictly positive parameters $\left(\gamma_{n}\right)_{n \in \mathbb{N}}$ and a starting point $x_{0}$, the inductive update rule of the Proximal Point Algorithm for minimizing $\varphi$ is

$$
(\forall n \in \mathbb{N}) \quad x_{n+1}=\underset{x \in X}{\arg \min }\left[\varphi(x)+\frac{1}{2 \gamma_{n}}\left\|x-x_{n}\right\|^{2}\right] .
$$

The point $x_{n+1}$ appearing in the update rule (3.1) is uniquely determined: using the sum rule [16, Theorem 28.2], we have $0 \in \partial\left(\varphi+\frac{1}{2 \gamma_{n}}\left\|\cdot-x_{n}\right\|^{2}\right)\left(x_{n+1}\right)=\partial \varphi\left(x_{n+1}\right)+$ $\left(x_{n+1}-x_{n}\right) / \gamma_{n}$; equivalently,

$$
x_{n} \in\left(I+\gamma_{n} \partial \varphi\right)\left(x_{n+1}\right)
$$

or

$$
x_{n+1}=\left(I+\gamma_{n} \partial \varphi\right)^{-1}\left(x_{n}\right) .
$$

A classical result due to Minty [12] states that the operator $\left(I+\gamma_{n} \partial \varphi\right)^{-1}$ appearing in (3.3), which is also known as the proximal mapping [13] or as the resolvent of the maximal monotone operator $\partial \varphi$, is single-valued (in fact, firmly nonexpansive) 
and everywhere defined. Thus the update rule (3.1) uniquely determines the next iterate.

We now show that the Support Point Algorithm is a special case of the Proximal Point Algorithm.

Theorem 3.1. Suppose $C$ is a closed convex nonempty set in $X$ and $f \in X$ such that $\|f\|=1$. Then the Support Point Algorithm with respect to $f$ and $C$ (see Theorem [2.5) is precisely the Proximal Point Algorithm for minimizing $-f+\iota_{C}$ (see Section (1) if both methods employ the same starting point and the same parameter sequence.

Proof. Fix a common starting point $x_{0}$ and a common parameter sequence $\left(\alpha_{n}\right)_{n \in \mathbb{N}}$. Set $\varphi=-f+\iota_{C}$. The sum rule for subdifferentials [16] Theorem 28.2] implies $\partial \varphi=-f+N_{C}$. The (implicit) update rule for the Proximal Point Algorithm (3.2) thus becomes $x_{n} \in\left(I+\alpha_{n} \partial \varphi\right)\left(x_{n+1}\right)=x_{n+1}-\alpha_{n} f+\alpha_{n} N_{C}\left(x_{n+1}\right)=x_{n+1}-\alpha_{n} f+$ $N_{C}\left(x_{n+1}\right) \Leftrightarrow x_{n}+\alpha_{n} f \in\left(I+N_{C}\right)\left(x_{n+1}\right) \Leftrightarrow x_{n+1}=\left(I+N_{C}\right)^{-1}\left(x_{n}+\alpha_{n} f\right)=$ $P_{C}\left(x_{n}+\alpha_{n} f\right)$ (using Proposition [2.3(iii)), which is precisely the update rule for the Support Point Algorithm.

\section{Description of Hundal's alternating projections example}

Let $H$ and $K$ be two closed convex sets in $X$ with $H \cap K \neq \varnothing$. Given an arbitrary starting point $y_{0} \in X$, the Method of Alternating Projections aims to find a point in $H \cap K$ iteratively through the sequence $\left(y_{n}\right)_{n \in \mathbb{N}}$ generated by

$$
(\forall n \in \mathbb{N}) \quad y_{n+1}=P_{K} P_{H} y_{n}
$$

This algorithm goes back at least to 1933 when von Neumann demonstrated that $y_{n} \rightarrow P_{H \cap K} y_{0}$ provided that $H$ and $K$ are two subspaces; see [8, Chapter 9] for further information. In 1965, Bregman [6] proved that, in general, the sequence $\left(y_{n}\right)_{n \in \mathbb{N}}$ converges weakly to some point in $H \cap K$. Since then, the obvious question was whether the convergence is actually strong. Hundal recently answered this question in the negative [10]:

Example 4.1 (Hundal). [10. Suppose $X=\ell_{2}$. Then there exist two closed convex sets $H, K$ in $X$, a point $f \in X$, and a point $y_{0} \in K$ such that the following holds:

(i) $\|f\|=1$;

(ii) $H$ is a hyperplane, represented as $H=\{f\}^{\perp}$;

(iii) $K$ is a cone and $\sup \langle f, K\rangle=0$;

(iv) $H \cap K=\{0\}$;

(v) the sequence $\left(y_{n}\right)_{n \in \mathbb{N}}=\left(\left(P_{K} P_{H}\right)^{n} y_{0}\right)_{n \in \mathbb{N}}$ converges weakly to 0 , but not in norm.

Hundal's ingenious construction, while lengthy and quite technical, is entirely elementary in the sense that it does not rely upon deeper results from other areas. The normal vector $f$ of the hyperplane $H$ is simply the negative of the first standard unit vector; however, the cone $K$ is more complicated. (For instance, in view of [3] Theorem 5.3], $K$ fails to be a lattice cone.)

\section{NeW Proximal Point iteration that DOES NOT CONVERGE IN NORM}

Theorem 5.1. Let $H, K, f$, and $\left(y_{n}\right)_{n \in \mathbb{N}}$ be as in Example 4.1. Then $\left(y_{n}\right)_{n \in \mathbb{N}}$ is generated by the Support Point Algorithm with respect to $f$ and $K$, with starting 
point $y_{0}$ and strictly positive parameter sequence $\left(\alpha_{n}\right)_{n \in \mathbb{N}}=\left(\left|\left\langle f, y_{n}\right\rangle\right|\right)_{n \in \mathbb{N}}$. Moreover, $\left(\alpha_{n}\right)_{n \in \mathbb{N}}$ is decreasing, $\sum_{n \in \mathbb{N}} \alpha_{n}=+\infty$, and $\sum_{n \in \mathbb{N}} \alpha_{n}^{2}<+\infty$. In particular, the sequence $\left(y_{n}\right)_{n \in \mathbb{N}}$ is an iteration of the Proximal Point Algorithm that converges weakly, but not in norm.

Proof. Write $S=\operatorname{supp}(f, K)$.

Claim 1: $S=\{0\}$.

On the one hand, by Example 4.1.(iii), $0 \in S$. On the other hand, pick $x \in S$. Then $\langle f, x\rangle=\langle f, 0\rangle=0$. Hence $x \in\{f\}^{\perp}=H$, and thus $x \in S \cap H \subseteq K \cap H=\{0\}$ (Example 4.1(iv)). Altogether, $S=\{0\}$.

Claim 2: $(\forall n \in \mathbb{N}) \alpha_{n}=\left\|y_{n}-P_{H} y_{n}\right\|>0$ and $P_{H} y_{n}=y_{n}+\alpha_{n} f$.

In view of Example 4.1.(iv) \&(v), $\left(y_{n}\right)_{n \in \mathbb{N}}$ lies in $K \backslash H=K \backslash\{0\}$, and $\left(P_{H} y_{n}\right)_{n \in \mathbb{N}}$ belongs to $H \backslash K=H \backslash\{0\}$. Fix $n \in \mathbb{N}$. The well-known formula for the projection onto a hyperplane [8, Section 6.17] now yields $y_{n} \neq P_{H} y_{n}=y_{n}-\left\langle f, y_{n}\right\rangle f=y_{n}+$ $\left|\left\langle f, y_{n}\right\rangle\right| f$ and $0<\left\|y_{n}-P_{H} y_{n}\right\|=\left|\left\langle f, y_{n}\right\rangle\right|=\alpha_{n}$ (recall $\|f\|=1$ by Example 4.1.(i) and $\left\langle f, y_{n}\right\rangle<0$ by Example 4.1.(iii)\&(iv)).

Claim 3: The sequence $\left(y_{n}\right)$ is generated by the Support Point Algorithm with respect to $f$ and $K$, with starting point $y_{0}$ and parameter sequence $\left(\alpha_{n}\right)_{n \in \mathbb{N}}$.

In view of Claim 2, the update rule becomes $(\forall n \in \mathbb{N}) y_{n+1}=P_{K} P_{H} y_{n}=$ $P_{K}\left(y_{n}+\alpha_{n} f\right)$. Since each $\alpha_{n}$ is strictly positive (Claim 2), this claim is verified as well.

Claim 4: $\left(\alpha_{n}\right)_{n \in \mathbb{N}}$ is decreasing.

Fix $n \in \mathbb{N}$. Using Claim 2, the definition of the sequence $\left(y_{n}\right)_{n \in \mathbb{N}}$ and the fact that it belongs to $K$ (Example4.1), and the definition of the distance function (Definition [2.2), we obtain $\alpha_{n}=\left\|y_{n}-P_{H} y_{n}\right\| \geq d\left(P_{H} y_{n}, K\right)=\left\|P_{K} P_{H} y_{n}-P_{H} y_{n}\right\|=$ $\left\|y_{n+1}-P_{H} y_{n}\right\| \geq d\left(y_{n+1}, H\right)=\left\|y_{n+1}-P_{H} y_{n+1}\right\|=\alpha_{n+1}$, as desired.

Claim 5: $\sum_{n \in \mathbb{N}} \alpha_{n}=+\infty$.

If $\sum_{n \in \mathbb{N}} \alpha_{n}<+\infty$, then $\left(y_{n}\right)_{n \in \mathbb{N}}$ would converge in norm (Claim 3 and Theorem [2.5. (i)), which contradicts Example 4.1. (v).

Claim 6: $\sum_{n \in \mathbb{N}} \alpha_{n}^{2}<+\infty$.

Fix $n \in \mathbb{N}$. Then Proposition 2.3 (ii), $H \cap K=\{0\}$ (Example 4.1(iv)), and Claim 2 yield

$$
\begin{aligned}
\left\|y_{n}\right\|^{2} & =\left\|y_{n}-0\right\|^{2} \geq\left\|P_{H} y_{n}-P_{H} 0\right\|^{2}+\left\|y_{n}-P_{H} y_{n}\right\|^{2} \\
& =\left\|P_{H} y_{n}-0\right\|^{2}+\alpha_{n}^{2} \geq\left\|P_{K} P_{H} y_{n}-P_{K} 0\right\|^{2}+\alpha_{n}^{2}=\left\|y_{n+1}\right\|^{2}+\alpha_{n}^{2} .
\end{aligned}
$$

Hence $\left\|y_{n}\right\|^{2}-\left\|y_{n+1}\right\|^{2} \geq \alpha_{n}^{2}$, and thus $\left\|y_{0}\right\|^{2} \geq \sum_{n \in \mathbb{N}} \alpha_{n}^{2}$.

Claim 7: $\left(y_{n}\right)_{n \in \mathbb{N}}$ is a proximal point iteration that converges weakly, but not in norm.

This is clear from Claim 3 and Theorem 3.1

The entire theorem is proven.

Remark 5.2. For another iteration of the proximal point algorithm that converges weakly but not in norm, also based on Hundal's alternating projections example, we refer the reader to [5].

\section{ACKNOWLEDGMENT}

We thank Patrick Combettes and an anonymous referee for careful reading and helpful comments. 


\section{REFERENCES}

[1] H. H. Bauschke, Projection Algorithms and Monotone Operators, Ph.D. Thesis, Department of Mathematics and Statistics, Simon Fraser University, Burnaby, British Columbia, August 1996. Available at http://www.cecm.sfu.ca/preprints/1996pp.html.

[2] H. H. Bauschke and J. M. Borwein, On projection algorithms for solving convex feasibility problems, SIAM Rev., 38 (1996), 367-426. MR.1409591 (98f:90045)

[3] H. H. Bauschke and J. M. Borwein, On the Convergence of the von Neumann's Alternating Projection Algorithm for Two Sets, Set-Valued Anal., 1 (1993), 185-212. MR1239403 (95d:65048)

[4] H. H. Bauschke and P. L. Combettes, A weak-to-strong convergence principle for Fejérmonotone methods in Hilbert spaces, Math. Oper. Res., 26 (2001), 248-264. MR1895827 (2003f:65101)

[5] H. H. Bauschke, E. Matoušková, and S. Reich, Projection and proximal point methods: convergence results and counterexamples, Nonlinear Anal., 56 (2004), 715-738. MR2036787 (2004m:47116)

[6] L. M. Bregman, The method of successive projection for finding a common point of convex sets, Soviet Math. Dokl., 6 (1965), 688-692.

[7] H. Brézis and P.-L. Lions, Produits infinis de résolvantes, Israel J. Math., 29 (1978), 329-345. MR 0491922 (80b:47068)

[8] F. Deutsch, Best Approximation in Inner Product Spaces, Springer-Verlag, New York, 2001. MR:1823556 (2002c:41001)

[9] O. Güler, On the convergence of the proximal point algorithm for convex minimization, SIAM J. Control Optim., 29 (1991), 403-419. MR1092735 (92c:90086)

[10] H. Hundal, An Alternating Projection that Does Not Converge in Norm, Nonlinear Anal., 57 (2004), 35-61. MR 2055986 (2005a:47093)

[11] B. Martinet, Régularisation d'inéquations variationnelles par approximations successives, Rev. Française Informat. Recherche Opérationnelle (Ser. R-3), 4 (1970), 154-158. MR0298899 (45:7948)

[12] G. Minty, Monotone (nonlinear) operators in a Hilbert space, Duke Math. J., 29 (1962), 341-346. MR0169064 (29:6319)

[13] J.-J. Moreau, Proximité et dualité dans un espace hilbertien, Bull. Soc. Math. France, 93 (1965), 273-299. MR0201952 (34:1829)

[14] R. R. Phelps, Convex functions, monotone operators and differentiability (Second Edition), Springer-Verlag, Berlin, 1993. MR,1238715 (94f:46055)

[15] R. T. Rockafellar, Monotone operators and the proximal point algorithm, SIAM J. Control Optim., 14 (1976), 877-898. MR.0410483 (53:14232)

[16] S. Simons, Minimax and Monotonicity, 1998. MR1723737|(2001h:49002)

[17] M. V. Solodov and B. F. Svaiter, Forcing strong convergence of proximal point iterations in a Hilbert space, Math. Program. (Ser. A), 87 (2000), 189-202. MR1734665 (2000j:90077)

Department of Mathematics and Statistics, University of Guelph, Guelph, Ontario, CANADA N1G 2W1

E-mail address: hbauschk@uoguelph.ca

Department of Mathematics, University of Washington, Box 354350, Seattle, WashINGTON 98195-4350

E-mail address: burke@math.washington.edu

Department of Mathematics, The Pennsylvania State University, University Park, PenNSYlVANia 16802

E-mail address: deutsch@math.psu.edu

Momentum Investment Services, 146 Cedar Ridge Drive, Port Matilda, Pennsylvania 16870

E-mail address: hundal@math.psu.edu

Department of Mathematics, La Sierra University, Riverside, California 92515

E-mail address: jvanderw@LaSierra.edu 